\title{
Transcatheter mitral valve repair/replacement for primary mitral regurgitation
}

\author{
Ted Feldman, Eugene Fernandes, Justin P. Levisay \\ NorthShore University HealthSystem, Evanston, IL, USA \\ Correspondence to: Ted Feldman, MD, FESC, FACC, MSCAI. Evanston Hospital, Cardiology Division-Walgreen Building 3rd Floor, 2650 Ridge \\ Ave., Evanston, IL, USA. Email: tfeldman@tfeldman.org.
}

\begin{abstract}
This review outlines the first trial experience with transcatheter therapy for mitral regurgitation (MR), developed from the EVEREST II MitraClip trial in a trial population comprised predominantly of patients with degenerative mitral regurgitation (DMR). Subsequent experience with MitraClip and several other devices has been mostly in functional MR patients. At the same time, there has been ongoing experience with MitraClip in DMR, and a variety of other devices have been developed for catheter-based treatment of MR. Annuloplasty devices have been indicated for DMR, and the potential for transcatheter annuloplasty to be used, in conjunction with other catheter techniques, such as chordal replacement, as it is in standard mitral repair, is developing. Transcatheter mitral valve replacement will clearly have some role for MR of both functional and degenerative etiologies, when repair is not feasible or fails. This review will discuss the evidence base and future development of these mitral repair and replacement approaches for DMR.
\end{abstract}

Keywords: Mitral regurgitation (MR); mitral repair; degenerative mitral regurgitation (DMR); percutaneous repair

Submitted May 14, 2018. Accepted for publication Jul 18, 2018.

doi: 10.21037/acs.2018.07.04

View this article at: http://dx.doi.org/10.21037/acs.2018.07.04

\section{Introduction}

The majority of trial and clinical patients managed with transcatheter therapy to date have functional mitral regurgitation (FMR) as the etiology of mitral regurgitation (MR). At the same time, the device with by far the largest application in clinical practice, the MitraClip, was originally targeted for degenerative mitral regurgitation (DMR) and is approved for use in the United States specifically and exclusively for this purpose. A role for catheter-based annuloplasty devices has similarly also been recognized for DMR. The potential for transcatheter annuloplasty to be used in conjunction with other catheter techniques, such as chordal replacement, as it is in standard mitral repair surgery, has promise. Transcatheter mitral valve replacement will clearly have some role for MR of both functional and degenerative etiologies, when repair is not feasible or fails.

\section{MitraClip for degenerative MR}

The initial efforts to treat DMR with catheter-based methods began with the MitraClip device, with the 1st human implant in 2003 (Figure 1). MitraClip was designed to mimic or recapitulate the surgical edge-to-edge repair (Figure 2). The surgical edge-to-edge repair was used almost exclusively in DMR. The initial efforts to utilize the MitraClip were targeted primarily towards DMR as well. Subsequently, the MitraClip device has been used in tens of thousands of patients worldwide, with the majority of use in FMR. Nevertheless, a great deal of experience has been developed with DMR. The majority of the published experience in DMR patients derives from the EVEREST trials.

The EVEREST II trial randomly assigned 279 patients with moderately severe or severe (grade $3+$ or $4+$ ) MR in a 2:1 ratio to undergo either percutaneous repair or conventional surgery for repair or replacement of the mitral valve (1). The primary composite end-point for efficacy was freedom from death, surgery for mitral-valve dysfunction and from grade $3+$ or $4+M R$ at 12 months. The primary safety end-point was a composite of major adverse events 

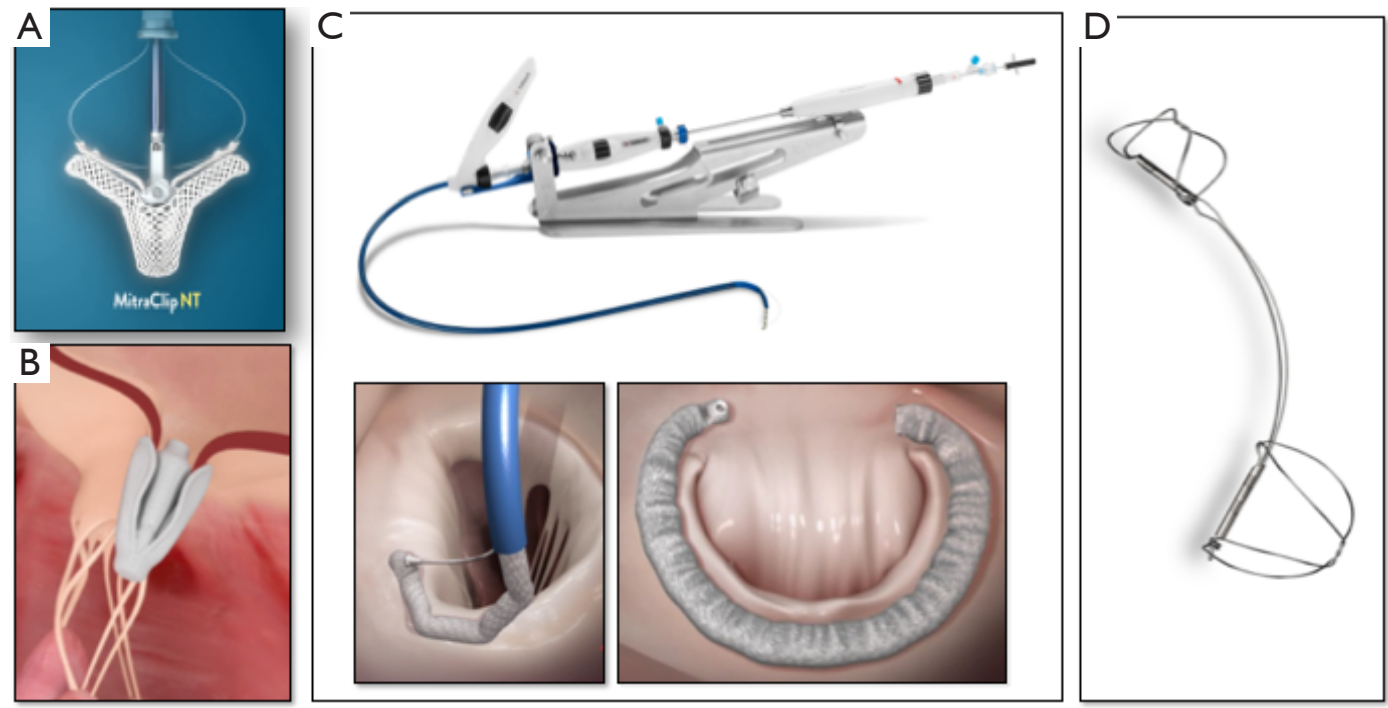

Figure 1 Mitral repair devices: (A) MitraClip NT for leaflet repair (Abbott Vascular, Santa Clara, CA, USA). (B) PASCAL for edge-toedge repair with a spacer and clasps (Edwards Lifesciences, Irvine, CA, USA). (C) Cardioband for transseptal direct annuloplasty (Edwards Lifesciences, Irvine, CA, USA). (D) Carillon for indirect annuloplasty via the coronary sinus (Cardiac Dimensions, Redmond, WA, USA).
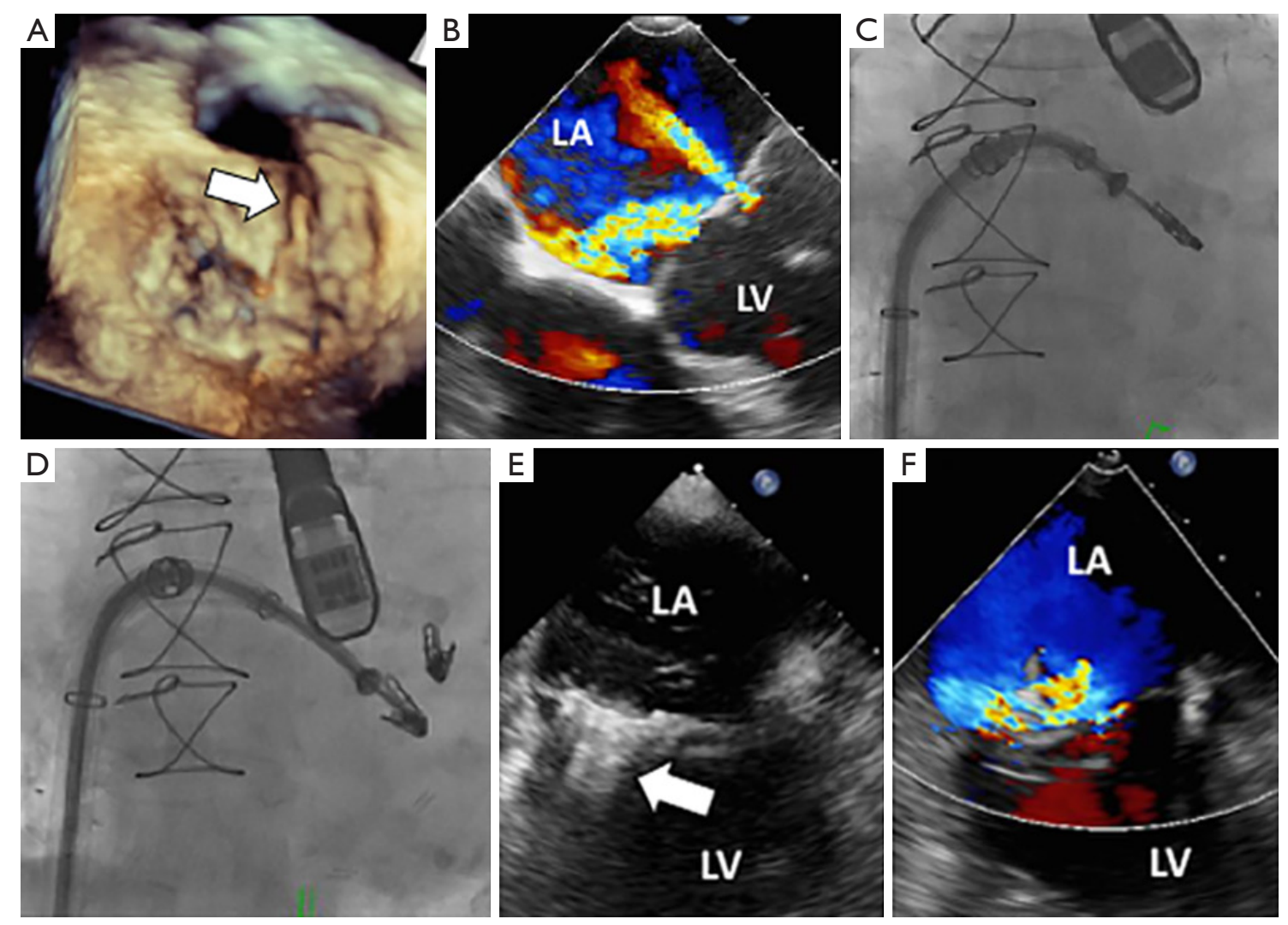

Figure 2 MitraClip case example. (A) 3D left atrial view of the mitral valve. The arrow indicates a ruptured chordae; (B) color Doppler showing severe MR prior to intervention; (C,D) fluoroscopic images of placement of two MitraClip devices; (E) echo appearance of the 2 clips; (F) color Doppler showing improved MR after intervention. LA, left atrium, LV, left ventricle. 
within 30 days. At 12 months, the rates of the primary endpoint for efficacy were $55 \%$ in the percutaneous repair group and $73 \%$ in the surgery group $(\mathrm{P}=0.007)$. The respective rates of the components of the primary endpoint were as follows: death, $6 \%$ in each group; surgery for mitral-valve dysfunction, $20 \%$ vs. $2 \%$; and grade $3+$ or $4+$ MR, $21 \%$ vs. $20 \%$. Major adverse events occurred in $15 \%$ of patients in the percutaneous repair group and $48 \%$ of patients in the surgery group at 30 days $(\mathrm{P}<0.001)$. At 12 months, both groups had improved left ventricular size, New York Heart Association functional class, and quality-of-life measures, as compared with baseline. This study concluded that although percutaneous repair was less effective at reducing MR than conventional surgery, the procedure was associated with superior safety and similar improvements in clinical outcomes. Patients were enrolled from September 2005 through November 2008, so the results reflect early experience, the learning curve for the procedure and for patient selection.

Many additional lessons were learned from the five-year results. At 5 years, the rate of the composite end-point of freedom from death, surgery, or $3+$ or $4+M R$ in the astreated population was $44.2 \%$ vs. $64.3 \%$ in the percutaneous repair and surgical groups, respectively $(\mathrm{P}<0.01)$. The difference was driven by increased rates of $3+$ to $4+M R$ $(12.3 \%$ vs. $1.8 \% ; \mathrm{P}<0.02)$ and surgery $(27.9 \%$ vs. $8.9 \%$; $\mathrm{P}<0.003)$ with percutaneous repair. Five-year mortality rates were $20.8 \%$ and $26.8 \%(\mathrm{P}<0.4)$ for percutaneous repair and surgery, respectively. In multivariate analysis, the treatment strategy was not associated with survival. After percutaneous repair, $78 \%$ of surgeries occurred within the first six months. Beyond six months, rates of surgery and moderate-to-severe MR were comparable between groups. This last point merits careful consideration.

Between 1- and 5-year follow-up, comparably low rates of surgery for MV dysfunction with either percutaneous or surgical therapy demonstrate the durability of MR reduction with both repair techniques (2). A landmark analysis showed virtually all of the failures for MitraClip occurred within the first six months after treatment. In the EVEREST II randomized trial, the procedure was in its infancy and all the operators were inexperienced. Single leaflet detachment occurred in almost $5 \%$ and there was failure to implant a device in $9 \%$ of patients. Almost one third of patients in EVEREST II had complex DMR with either Barlow's disease or anterior leaflet prolapse. In current practice, single leaflet detachment occurs in about $1 \%$ of cases and failure to implant a device is rare. Patient selection based on valve morphology has evolved and complex leaflet pathology is now treated with MitraClip only as a last resort (3). Thus, it might be speculated that if a randomized comparison of surgical and percutaneous repair were conducted today, the advantages of percutaneous repair might be amplified by the improved early procedure success that is typical in contemporary practice.

Another important finding from the 5 -year analysis of the EVEREST II Trial was the absence of annular dilatation in the MitraClip treated group. It was predicted that because almost half of the MitraClip patients have 2+ residual MR and since no annuloplasty was done in the MitraClip patients-based on prior surgical experienceprogressive LV and annular dilatation would be expected. In fact, there was persistent reduction in $\mathrm{LV}$ dimensions at 5 years. The five-year report supports the durability of MR reduction observed after successful device implantation, with resultant symptom alleviation and LV reverse remodeling. The findings endorse the long-term safety of this approach and refute concerns that greater residual MR after percutaneous repair results in progressive $\mathrm{MR}$, annular dilatation, or reduced long-term survival.

During the conduct of the EVEREST II randomized comparison with surgery, it became clear that many of the patients that were being referred for consideration for the trial were not candidates for surgery, and thus could not be randomized. This experience was consistent with observations that a large proportion of patients with MR were never referred for surgery, in part because less invasive therapy options were not available $(4,5)$. Accordingly, a registry was created for high risk patients (6). The enrolled patients were selected with the same anatomic criteria used for the randomized EVEREST II trial. Patients with grades $3+$ to $4+M R$ and a surgical mortality risk of $\geq 12 \%$, based on the Society of Thoracic Surgeons risk calculator or the estimate of a surgeon coinvestigator following pre-specified protocol criteria, were enrolled. Three hundred and fifty-one patients completed 12 months of follow-up. Patients were elderly ( $76 \pm 11$ years of age), with $70 \%$ having functional MR and $60 \%$ having prior cardiac surgery. MitraClip reduced MR to $\leq 2+$ in $86 \%$ of patients at discharge. Major adverse events at 30 days included death in $4.8 \%$, myocardial infarction in $1.1 \%$, and stroke in $2.6 \%$. At 12 months, MR was $\leq 2+$ in $84 \%$ of patients. From baseline to 12 months, left ventricular enddiastolic volume improved from $161 \pm 56$ to $143 \pm 53 \mathrm{~mL}$ $(\mathrm{P}<0.0001)$ and $\mathrm{LV}$ end-systolic volume improved from $87 \pm 47$ to $79 \pm 44 \mathrm{~mL}(\mathrm{P}<0.0001)$. NYHA class improved 
from $82 \%$ with class III/IV at baseline to $83 \%$ with class I/II at 12 months $(\mathrm{P}<0.0001)$. The 36-item Short Form Health Survey physical and mental quality-of-life scores improved from baseline to 12 months (physical component score from $34.0 \pm 9.1$ to $38.8 \pm 11.3$ and mental component score from $44.9 \pm 13.5$ to $49.8 \pm 12.2, \mathrm{P}<0.0001)$. The annual hospitalization rate for heart failure fell from $0.79 \%$ preprocedure to $0.41 \%$ post-procedure $(\mathrm{P}<0.0001)$. The Kaplan-Meier survival estimate at 12 months was $77.2 \%$. The percutaneous mitral valve device significantly reduced MR, improved clinical symptoms, and decreased LV dimensions at 12 months in this high-surgical-risk cohort. Although most of these patients had FMR, outcomes did not differ between DMR and FMR etiologies.

A prohibitive-risk DMR cohort from the high-risk registry was identified by a multidisciplinary heart team that retrospectively evaluated high-risk DMR patients enrolled in the EVEREST studies. A total of 141 high-risk DMR patients were consecutively enrolled; 127 were identified as meeting the definition of prohibitive risk and had 1-year follow-up (median: 1.47 years) available (7). Patients were elderly (mean age: 82.4 years), severely symptomatic (87\% New York Heart Association class III/IV) and were at prohibitive surgical risk (STS score: $13.2 \% \pm 7.3 \%$ ). The MitraClip procedure was successfully performed in $95.3 \%$; hospital stay was $2.9 \pm 3.1$ days. Major adverse events at 30 days included death in $6.3 \%$, myocardial infarction in $0.8 \%$, and stroke in $2.4 \%$. Through 1 year, there were a total of 30 deaths (23.6\%), with no survival difference between patients discharged with $M R \leq 1+$ or $M R 2+$. At 1 year, the majority of surviving patients $(82.9 \%)$ remained MR $\leq 2+$ at 1 year, and $86.9 \%$ were in NYHA class I or II. Left ventricular end-diastolic volume decreased (from $125.1 \pm 40.1$ to $108.5 \pm 37.9 \mathrm{~mL} ; \mathrm{P}<0.0001)$. SF-36 quality-oflife scores improved and hospitalizations for heart failure were reduced in patients whose MR was reduced. MitraClip in prohibitive surgical risk patients was associated with safety and good clinical outcomes, including decreases in re-hospitalization, functional improvements, and favorable ventricular remodeling at 1 year. This analysis, in conjunction with the EVEREST II randomized trial and the high-risk registry, led to FDA approval of the MitraClip for patients with symptomatic DMR deemed high risk for mitral valve surgery on Oct 25, 2013.

Since FDA approval, there has been significant use of MitraClip for the DMR indication in the United States. Post-market surveillance has been required. The commercial experience with MitraClip has been collected by the Society of Thoracic Surgery/American College of Cardiology Transcatheter Valve Therapy Registry. Commercially treated patients from the registry were recently described (8). The population consisted of 2,952 patients treated at 145 hospitals between November 2013 and September 2015. In 1,867 patients, data were linked to patient-specific Centers for Medicare and Medicaid Services administrative claims for analyses. The median age was 82 years $(55.8 \%$ men), with a median Society of Thoracic Surgery predicted risk of mortality of $6.1 \%$ for mitral repair (interquartile range: $3.7 \%$ to $9.9 \%$ ) and $9.2 \%$ (interquartile range: $6.0 \%$ to $14.1 \%$ ) for replacement. Overall, inhospital mortality was $2.7 \%$. Acute procedure success occurred in $91.8 \%$. Among the patients with Centers for Medicare and Medicaid Services linkage data, the mortality at 30 days and at one year was $5.2 \%$ and $25.8 \%$, respectively, and repeat hospitalization for heart failure at one year occurred in $20.2 \%$. The variables associated with mortality or rehospitalization for heart failure after multivariate adjustment were increasing age, lower baseline left ventricular ejection fraction, poorer post-procedural MR, moderate or severe lung disease, dialysis, and severe tricuspid regurgitation. The findings demonstrate that commercial transcatheter mitral valve repair is being performed in the United States with acute effectiveness and safety. The one-year mortality of $25.8 \%$ is high, reflecting the high-risk profile of these patients.

Further outcomes from several registry studies have been summarized in a meta-analysis (9). They are concordant, overall, with the EVEREST prospective registries. A total of nine studies with 2,615 patients with functional and degenerative MR were included. At oneyear, there were no significant differences among groups in terms of patients with $M R$ grade $\leq 2$, while there was a significantly lower rate of mitral valve re-intervention in patients with FMR compared with those with DMR (4\% vs. 10\%; RR 0.60; 95\% CI: 0.38 to $0.97 ; \mathrm{P}=0.04)$. One-year mortality rate was $16 \%$ and similar among groups. FMR patients showed significantly higher percentage of patients in NYHA class III/IV (16\% vs. $8 \% ; \mathrm{P}<0.01)$ and re-hospitalization for heart failure $(23 \%$ vs. $14 \%$; $\mathrm{P}=0.03)$. This meta-analysis suggests that percutaneous edge-to-edge repair is efficacious and safe in patients with both functional and degenerative MR.

A new device for percutaneous edge-to-edge repair has recently been described. The novel Edwards PASCAL transcatheter mitral valve repair system (Edwards Lifesciences, Irvine, CA, USA) addresses several of the limitations of MitraClip by simplifying navigation in 
the left atrium, improving reduction of MR through implementation of a central spacer and by allowing for independent leaflet grasping. 30-day results of a firstin-man study have been reported (10). Additionally, a multicenter, prospective, observational, compassionate use program enrolled patients with symptomatic, severe functional, degenerative, or mixed MR deemed at high risk, or surgically-prohibitive risk. 23 patients (median age 75 years) had treatment for grade $3+$ to $4+M R$. The median EuroSCORE II score was $7.1 \%$ and the median Society of Thoracic Surgeons predicted risk of mortality for mitral valve repair was $4.8 \%$ and $6.8 \%$ for mitral replacement. The implantation of at least one device was successful in all patients, resulting in procedural residual MR of grade 2+ or less in $96 \%$ of the subjects. $26 \%$ of the cohort had two implants. Periprocedural complications occurred in two of 23 patients (one minor bleeding event and one transient ischemic attack). Despite the anatomical complexity of MR in this compassionate use cohort, technical success was achieved in $96 \%$. Three patients (13\%) died during the 30 -day follow-up. $95 \%$ of patients were alive 30 days after implantation and were NYHA class I or II. An early feasibility trial is underway. The CLASP Study Edwards PASCAL TrAnScatheter Mitral Valve RePair System Study (CLASP) will enroll up to 120 patients in a multicenter, prospective single arm study (11). The primary outcome measures include a composite of major adverse events, defined as cardiovascular mortality, stroke, myocardial infarction, new need for renal replacement therapy, severe bleeding and re-intervention for study device-related complications at 30 days. The secondary outcome measures are $M R$ reduction, all-cause mortality, recurrent heart failure hospitalization and change in Six Minute Walk Test distance to 1 year.

The EVEREST II randomized trial completed enrollment in 2008. Since then, patient selection and procedural techniques have improved significantly. A contemporary randomized comparison of MitraClip and conventional mitral repair surgery for DMR is underway in France to assess the utility of percutaneous leaflet repair compared to surgery (12). The Multicenter Study of MITRACLIP Transcatheter Mitral Valve Repair in Patients with Severe Primary Mitral Regurgitation Eligible for High-risk Surgery (MITRA-HR) trial has a target enrollment of 330 patients to be randomized to the MitraClip NT System, compared to cardiac surgery with mitral valve repair or replacement. The inclusion criteria are: primary Mitral Regurgitation grade 3+ or 4+; NYHA class II to IV; mitral valve anatomy appropriate to MitraClip therapy; mitral valve surgery (repair or replacement); mitral valve area $\geq 3 \mathrm{~cm}^{2}$; minimal calcification in the grasping area; no leaflet cleft in the grasping area; flail width $<15$ $\mathrm{mm}$, and; flail gap $<10 \mathrm{~mm}$. Adult patients judged eligible for mitral valve surgery by the local heart team, but at high surgical risk, defined as age $\geq 75$ years and STS score $\geq 6 \%$ or one frailty index or major organ system compromise or one possible procedure specific impediment using MVARC definitions, or age $<75$ years and STS score $>8 \%$ or at least one other high-risk criterion following the MVARC definitions. The primary outcome measures include allcause mortality, unplanned hospitalizations for heart failure and mitral valve re-intervention. Secondary outcome measures include the occurrence of major adverse events at 30 days, including all-cause death, need for non-elective cardiovascular or thoracic surgery, device or procedurerelated adverse events, major bleeding complications or serious bleeding, major access site vascular complications, major cardiac structural complications, pulmonary complications (i.e., device or procedure-related), stroke and other cerebrovascular events, myocardial infarction, acute kidney injury or progression of chronic kidney disease, arrhythmias and conduction system disturbances, unplanned mitral valve surgery due to device/procedure failure or malfunction, requirement for valve replacement after valve repair failure and unplanned cardiac surgery for any cause. As of this writing in April 2018, the first patients have been enrolled in the trial. This trial could have a great impact on patient selection for MitraClip therapy for DMR, with the potential to change the indications to include high-risk patients who are currently surgical candidates.

\section{Annuloplasty}

Several percutaneous annuloplasty devices have been used in trials and clinical practice (Figure 1). Coronary sinus annuloplasty, an incomplete annuloplasty ring and complete rings have been used. The majority of the experience with these devices is in patients with FMR and the total number patients treated with all of these devices together is probably less than 1,500 , compared to the experience with MitraClip in over 60,000 cases. It is unlikely that transcatheter annuloplasty devices will be used alone in trials for DMR. For DMR, some of the most interesting applications of annuloplasty have been in conjunction with other catheterbased therapy approaches. The use of these devices in combination may mimic the routine use of multiple 

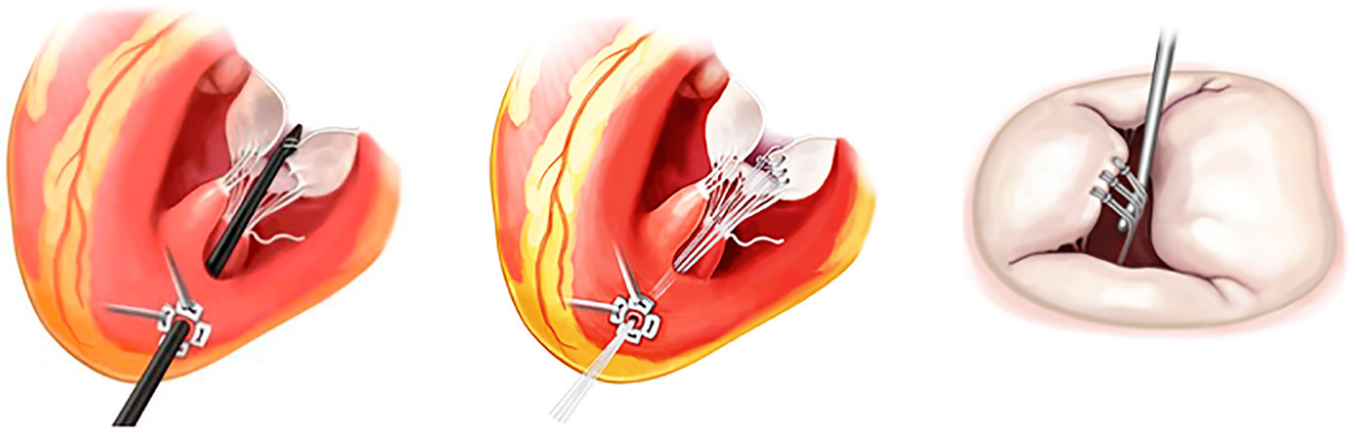

Figure 3 The transapical NeoChord repair procedure is dome under general anesthesia with trans esophageal guidance. Access is through a left lateral mini-thoracotomy. Purse-string sutures are placed postero-lateral from the LV apex. The NeoChord DS1000 device (NeoChord, Inc., St. Louis Park, MN, USA) is inserted into the LV and TEE is used to guide the device to the leaflet and implant the neochordae. When all neochordae are placed, they are tensioned under direct TEE visualization. The tensioned neochordae are secured to the LV epicardium using Teflon felt pledgets.

techniques in surgery for DMR, such as annuloplasty with either chordal replacement, or edge-to-edge repair.

One case report describes the use of the Carillon coronary sinus annuloplasty device in conjunction with MitraClip (13). The authors describe an 84-year-old female with EuroSCORE II: 9.64\% and severe DMR. The HeartTeam decision was made for a combined interventional approach mimicking the original Alfieri technique, which included annuloplasty and edge-to-edge plication of the mitral leaflets, using the Carillon-device and TMVR in combination with the MitraClip-system. The Carillondevice was placed on the posterior circumference of the mitral annulus, with reduction of MV annular diameters and no relevant effect on MR severity. MR reduction was achieved after placement of two clips with an acceptable mean pressure gradient of $5 \mathrm{mmHg}$. The procedure was completed in 80 minutes $(12.3 \mathrm{~min}$ radiation time, $90 \mathrm{~mL}$ contrast dye) without peri-procedural complications. The patient was discharged on day 7 after the procedure in good clinical condition and with NYHA class I. The authors concluded that 'untreatable' patients with mitral annular dilatation or other adverse anatomical features might benefit from approaching different pathologies of the mitral apparatus.

Another case report illustrates the results of combination therapy with transcatheter ring annuloplasty and neochord leaflet implantation (14). In mitral prolapse with flail leaflet, the combination of surgical repair including annuloplasty and chordal replacement is the current standard of care. They described a first-in-human case of a transvenous transcatheter off-pump direct annuloplasty with the
Edwards Cardioband system and simultaneous one-staged transapical off-pump transcatheter implantation of three NeoChordae using the NeoChord DS1000 application system to treat a 63-year-old male with an STS-predicted risk of mortality of $8 \%$.

\section{Chordal replacement}

Less invasive transapical chordal replacement has seen a significant development in experience worldwide. The largest experience is with the NeoChord device (NeoChord, Inc., St. Louis Park, Mn). The NeoChord DS 1000 System was CE marked and approved for sale in Europe in December 2012 (Figure 3). The largest experience with 1-year follow-up was recently published (15). Between 2013 and 2016, 213 patients were enrolled in the NeoChord Independent International Registry. All had severe MR due to flail or prolapse of 1 or both leaflets. The primary endpoint was composed of procedural success, freedom from mortality, stroke, reintervention, recurrence of severe $M R$, rehospitalization and decrease of $\geq$ NYHA class at 1 -year follow-up. The median age was 68 years and the median EuroSCORE II was $1.05 \%$. Procedural success was achieved in $96.7 \%$ patients. At 1 -year, overall survival was $98 \% \pm 1 \%$. Composite end point was achieved in $84 \% \pm 2.5 \%$ for the overall population, and for those with complex disease defined as anterior, bileaflet, paracommissural disease with/without leaflet/annular calcifications $63.6 \% \pm 8.4 \%(\mathrm{P}<0.0001)$. Clinical and echocardiographic efficacy is maintained up to 1 year with significant differences among the anatomical groups. Specific 

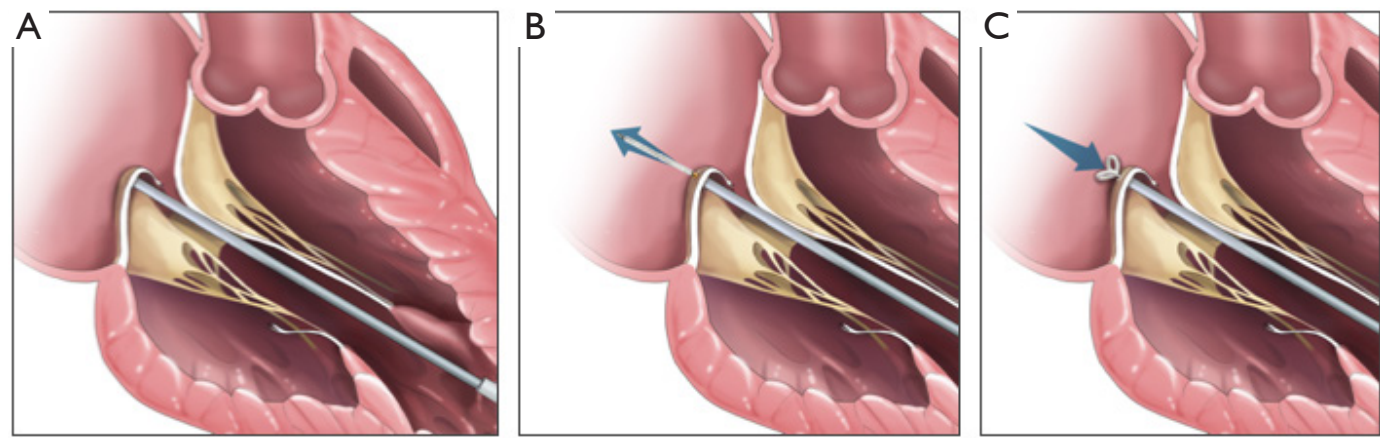

Figure 4 The procedure is performed under general endotracheal anesthesia with trans esophageal echo guidance, via a small thoracotomy. TEE is used to ensure that the entrance to the left ventricle apical to the base of the anterolateral papillary muscle. The delivery system is then inserted through the introducer and guided the prolapsed segment of the mitral leaflet using TEE. The end effector on the delivery system is used to stabilize the leaflet (A), and the pre-formed knot is deployed (B and C). After each knot, the delivery system is withdrawn, leaving 2 ePTFE strands exteriorized through the introducer. One delivery system is used for each knot. This sequence is repeated for the desired number of knots, usually 4 to 6 . The suture pairs are threaded individually through an ePTFE pledget and are tightened simultaneously using TEE guidance to minimize MR (Edwards Lifesciences, Irvine, CA, USA).

anatomical selection criteria are necessary to achieve stable results, with poorer outcomes in more complex leaflet anatomies.

The NeoChord system has also been used to create an edge-to-edge repair in a single case (16). The patient was treated as a bailout procedure. The 72-year-old male patient was considered high-risk (EuroSCORE II of 6.7\%) and frail and was not considered eligible for any other approved transcatheter therapy. The one-month outcome was favorable.

A critical trial is currently enrolling patients. The Randomized Trial of the NeoChord DS1000 System Versus Open Surgical Repair (ReChord) is underway in the United Sates (17). The target enrollment is 585 subjects, comparing the NeoChord trans-apical system with open surgical mitral repair in DMR. The primary outcome measures are the proportion of subjects free of major adverse events in the treatment group when compared to subjects in the control group at 30 days and the proportion of subjects free of MR grade $2-4+$, mitral valve replacement or mitral valve reintervention in the treatment group when compared to subjects in the control group at 1 year.

Another chordal replacement system has also been used in patients (Figure 4). The HARPOON device (Edwards Lifesciences Corporation, Irvine CA) has been evaluated in a prospective multicenter study (18). The TRACER (Mitral TransApical NeoChordal Echo-Guided Repair-CE Mark Study for the Harpoon Medical Device; NCT02768870) trial is a prospective, nonrandomized, multicenter clinical study designed to test the safety and performance of the Harpoon mitral valve repair system (Harpoon Medical Inc., Baltimore, Maryland). The system consists of 2 parts, namely a hemostatic $14 \mathrm{~F}$ introducer and a delivery system. The delivery system contains a pre-formed ePTFE knot that anchors artificial ePTFE cords on targeted locations on a prolapsed mitral leaflet. The procedure is done via a small thoracotomy. The delivery system is guided to the target on the prolapsed segment of the mitral leaflet using TEE guidance, and the knot is deployed. After each knot deployment, the delivery system is withdrawn from the heart, leaving 2 ePTFE strands exteriorized. This sequence is repeated for the desired number of knots, usually 4 to 6 times. The ePTFE suture pairs are threaded through a stiff ePTFE pledget and are tightened simultaneously and incrementally by using TEE guidance to optimize coaptation and minimize MR. Thirty patients with severe DMR were treated. The primary endpoint of successful implantation of cords with MR reduction to moderate or less was met in 27 of 30 patients (90\%). Three patients required conversion to open mitral surgery. There were no deaths, strokes, or permanent pacemaker implantations. At 1 month, MR was mild or less in $89 \%$ and was moderate in $11 \%$. At 6 months, MR was mild or less in $85 \%$ (22 of 26), moderate in 8\% (2 of 26 ) and severe in $8 \%$ ( 2 of 26). Favorable cardiac remodeling at 6 months included decreases in end-diastolic $(161 \pm 36$ to $122 \pm 30 \mathrm{~mL}$; $\mathrm{P}<0.001)$ and left atrial volumes $(106 \pm 36$ to $69 \pm 24 \mathrm{~mL}$; $\mathrm{P}<0.001)$. The anterior-posterior mitral annular dimension 


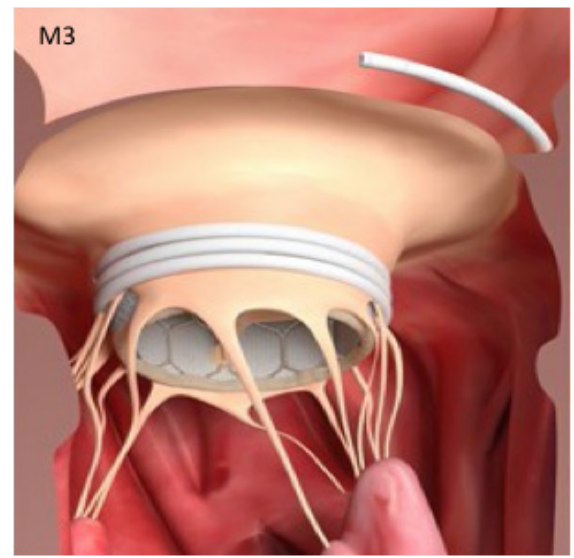

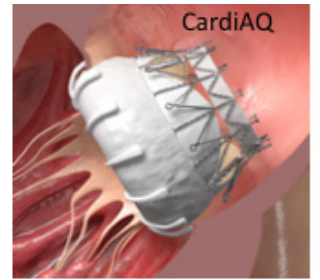
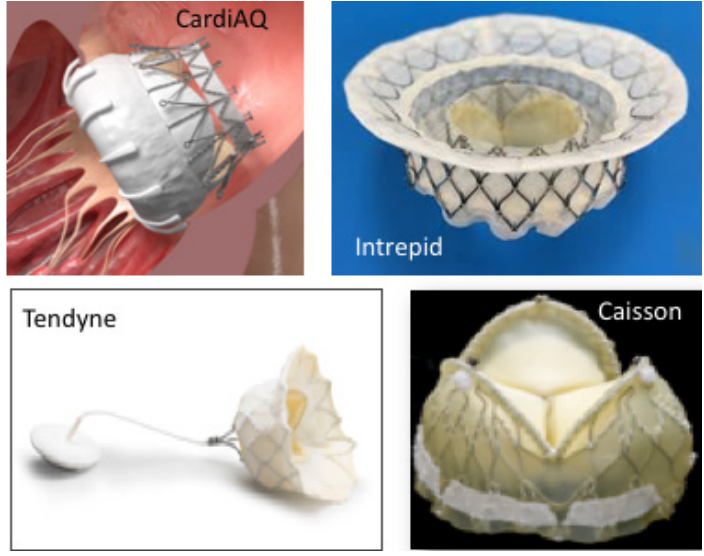

Figure 5 Transcatheter mitral valve devices in US Early Feasibility Trials (EFS). Left, M3 system (Edwards Lifesciences, Irvine, CA, USA), comprised of a helical docking wire that encircles the chordae, with a Sapien 3 valve implanted within the mitral leaflets. The CardiAQ valve is a placed via transseptal access (Edwards Lifesciences, Irvine, CA, USA). The Intrepid valve (Medtronic. Dublin, Ireland) is implanted via apical access, with a transseptal system under development. The Tendyne valve (Abbott Vascular, Santa Clara, CA, USA) is implanted via apical access, and has a tether that is anchored on the apical left ventricular epicardial surface. The Caisson valve (LivaNova, Maple Grove $\mathrm{MN}$ ) has a two-part system comprised of an annular frame, into which the tissue valve is implanted, all by transseptal approach. Other mitral devices in EFS trials include the Navigate (NaviGate Cardiac Structures Inc., Lake Forest CA) and Neovasc Tiarra (Neovasc Inc., Vancouver, B.C. Canada).

decreased $(34.7 \pm 5.8$ to $28.2 \pm 5.1 \mathrm{~mm}, \mathrm{P}<0.001)$ as did the mitral annular area $\left(10.0 \pm 2.7\right.$ vs. $\left.6.9 \pm 2.0 \mathrm{~cm}^{2} ; \mathrm{P}<0.0001\right)$. Further investigation with longer-term follow-up and direct comparison with conventional mitral valve surgery is necessary. The initial results are promising.

Historically, surgical repair procedures for DMR have routinely included annuloplasty in conjunction with other repair techniques, so much so that it has often been said that annuloplasty should always be part of a repair procedure. The development of techniques, such as less invasive chordal replacement, has led to growing recognition that annuloplasty is not always required, particularly when annular dilatation is not part of the mitral pathology.

\section{Transcatheter mitral valve replacement}

While there is still debate about the relative roles of repair and replacement for FMR, there is clear consensus and Guideline support for repair as the preferred approach for DMR. The majority of the development of transcatheter mitral valve replacement (TMVR) has been in the FMR population, and most of this has been transapical. This field is developing rapidly, with only a few hundred treated patients when all of the existing technologies are combined. There are eight TMVR devices in early feasibility trials in the United States, several of which are delivered via a transseptal approach (Figure 5). There will still clearly be a role for TMVR for DMR. One of the more frequent limiting factors for leaflet repair with MitraClip or PASCAL is a mitral valve area $<4 \mathrm{~cm}^{2}$. Leaflet calcification, short posterior leaflets, rheumatic etiology, Barlow's syndrome and clefts are additional unfavorable anatomic features for leaflet repair. These limitations will favor TMVR in some cases. Certainly, failed prior repair will also be an indication for TMVR.

\section{Acknowledgements}

None.

\section{Footnote}

Conflicts of Interest: Dr Feldman: Grants, consulting: Abbott, BSC, Edwards, WL Gore. Other authors have no conflicts of interest to declare.

\section{References}

1. Feldman T, Foster E, Glower DD, et al. Percutaneous repair or surgery for mitral regurgitation. $\mathrm{N}$ Engl J 
Med 2011;364:1395-406. Erratum in: N Engl J Med 2011;365:189.

2. Feldman T, Kar S, Elmariah S, et al. Randomized Comparison of Percutaneous Repair and Surgery for Mitral Regurgitation: 5-Year Results of EVEREST II. J Am Coll Cardiol 2015;66:2844-54.

3. Boekstegers $\mathrm{P}$, Hausleiter J, Baldus S, et al. Percutaneous interventional mitral regurgitation treatment using the Mitra-Clip system. Clin Res Cardiol 2014;103:85-96.

4. Lung B, Baron G, Butchart EG, et al. A prospective survey of patients with valvular heart disease in Europe: The Euro Heart Survey on Valvular Heart Disease. Eur Heart J 2003;24:1231-43.

5. Mirabel M, Lung B, Baron G, et al. What are the characteristics of patients with severe, symptomatic, mitral regurgitation who are denied surgery? Eur Heart J 2007;28:1358-65.

6. Glower DD, Kar S, Trento A, et al. Percutaneous mitral valve repair for mitral regurgitation in high-risk patients: results of the EVEREST II study. J Am Coll Cardiol 2014;64:172-81.

7. Lim DS, Reynolds MR, Feldman T, et al. Improved functional status and quality of life in prohibitive surgical risk patients with degenerative mitral regurgitation after transcatheter mitral valve repair. J Am Coll Cardiol 2014;64:182-92.

8. Sorajja P, Vemulapalli S, Feldman T, et al. Outcomes With Transcatheter Mitral Valve Repair in the United States: An STS/ACC TVT Registry Report. J Am Coll Cardiol 2017;70:2315-27.

9. Chiarito M, Pagnesi M, Martino EA, et al. Outcome after percutaneous edge-to-edge mitral repair for functional and degenerative mitral regurgitation: a systematic review and meta-analysis. Heart 2018;104:306-12.

10. Praz F, Spargias K, Chrissoheris M, et al. Compassionate use of the PASCAL transcatheter mitral valve repair system for patients with severe mitral regurgitation: a multicenter, prospective, observational, first-in-man study. Lancet 2017;390:773-80.

Cite this article as: Feldman T, Fernandes E, Levisay JP. Transcatheter mitral valve repair/replacement for primary mitral regurgitation. Ann Cardiothorac Surg 2018;7(6):755763. doi: $10.21037 /$ acs.2018.07.04
11. The CLASP Study Edwards PASCAL TrAnScatheter Mitral Valve RePair System Study (CLASP). Available online: https://clinicaltrials.gov/ct2/show/ NCT03170349?term=PASCAL\&rank=4. Accessed April 14, 2018.

12. Multicenter Study of MITRACLIP Transcatheter Mitral Valve Repair in Patients with Severe Primary Mitral Regurgitation Eligible for High-risk Surgery (MITRAHR). ClinicalTrials.gov Identifier: NCT03271762. Available online: https://clinicaltrials.gov/ct2/show/ NCT03271762? titles=MITRA-HR\&rank=1. Accessed April 14, 2018.

13. Schueler R, Werner N, Nickenig G, et al. Catheter-based complete "Alfieri-Stich" via interventional annuloplasty and edge-to-edge repair for degenerative mitral regurgitation. Eur Heart J 2016;37:2201.

14. von Bardeleben RS, Colli A, Schulz E, et al. First in human transcatheter $\mathrm{COMBO}$ mitral valve repair with direct ring annuloplasty and neochord leaflet implantation to treat degenerative mitral regurgitation: feasibility of the simultaneous toolbox concept guided by $3 \mathrm{D}$ echo and computed tomography fusion imaging. Eur Heart J 2018;39:1314-5.

15. Colli A, Manzan E, Aidietis A, et al. An early European experience with transapical off-pump mitral valve repair with NeoChord implantation. Eur J Cardiothorac Surg 2018;54:460-6.

16. Colli A, Besola L, Bizzotto E, et al. Edge-to-edge mitral valve repair with transapical neochord implantation. J Thorac Cardiovasc Surg 2018;156:144-8.

17. Randomized Trial of the Neochord DS1000 System Versus Open Surgical Repair (ReChord). Available online: https://clinicaltrials.gov/ct2/show/NCT02803957. Accessed April 14, 2018.

18. Gammie JS, Wilson P, Bartus K, et al. Response by Gammie et al to Letter Regarding Article, "Transapical Beating-Heart Mitral Valve Repair with an Expanded Polytetrafluoroethylene Chordal Implantation Device: Initial Clinical Experience". Circulation 2017;135:e18-9. 